\title{
INVARIANT EINSTEIN METRICS ON THREE-LOCALLY-SYMMETRIC SPACES
}

\author{
ZHIQI CHEN, YIFANG KANG, AND KE LIANG
}

\begin{abstract}
In this paper, we classify three-locally-symmetric spaces for a connected, compact and simple Lie group. Furthermore, we give the classification of invariant Einstein metrics on these spaces.
\end{abstract}

\section{INTRODUCTION}

A Riemannian manifold $(M,\langle\cdot, \cdot\rangle)$ is called Einstein if the Ricci tensor Ric of the metric $\langle\cdot, \cdot\rangle$ satisfies Ric $=c\langle\cdot, \cdot\rangle$ for some constant $c$. The above Einstein equation reduces to a system of nonlinear second-order partial differential equations. But it is difficult to get general existence results. Under the assumption that $M$ is a homogeneous Riemannian manifold, the Einstein equation reduces to a more manageable system of (nonlinear) polynomial equations, which in some cases can be solved explicitly. There are a lot of progress in the study on invariant Einstein metrics of homogeneous manifolds, such as the articles [1, 3, 4, 8, 9, 10, 15, 16, 17, 19, 20, 22, 23, 24, 25, 26, 29, 30, 31, 33, 34], and the survey article [28] and so on.

Consider a homogeneous compact space $G / H$ with a semisimple connected Lie group $G$ and a connected Lie subgroup $H$. Denote by $\mathfrak{g}, \mathfrak{h}$ the Lie algebras of $G, H$ respectively. Assume that $\mathfrak{p}$ is the orthogonal complement of $\mathfrak{h}$ in $\mathfrak{g}$ with respect to $B$, where $B$ is the Killing from of $\mathfrak{g}$. Every $G$-invariant metric on $G / H$ generates an adh-invariant inner product on $\mathfrak{p}$ and vice versa [7]. This makes it possible to identify invariant Riemannian metrics on $G / H$ with adh-invariant inner product on $\mathfrak{p}$. Note that the metric generated by the inner product $-\left.B\right|_{\mathfrak{p}}$ is called standard. Furthermore, if $G$ acts almost effectively on the homogeneous space $G / H$, and $\mathfrak{p}$ is the direct sum of three adh-invariant irreducible modules pairwise orthogonal with respect to $B$, i.e.,

$$
\mathfrak{p}=\mathfrak{p}_{1} \oplus \mathfrak{p}_{2} \oplus \mathfrak{p}_{3},
$$

with $\left[\mathfrak{p}_{i}, \mathfrak{p}_{i}\right] \subset \mathfrak{h}$ for any $i \in\{1,2,3\}$, then $G / H$ is called a three-locally-symmetric space.

The notation of a three-locally-symmetric space is introduced by Nikonorov in [26]. There has been a lot of study on invariant Einstein metrics for certain three-locally-symmetric spaces. For example, invariant Einstein metrics on the flag manifold $S U(3) / T_{\max }$ are given in [14], on

$$
S p(3) / S p(1) \times S p(1) \times S p(1) \text { and } F_{4} / \operatorname{Spin}(8)
$$

are obtained in [30], on the Kähler C-spaces

$S U\left(n_{1}+n_{2}+n_{3}\right) / S\left(U\left(n_{1}\right) \times U\left(n_{2}\right) \times U\left(n_{3}\right)\right), S O(2 n) / U(1) \times U(n-1), E_{6} / U(1) \times U(1) \times \operatorname{Spin}(8)$

are classified in [20, another approach to

$$
S U\left(n_{1}+n_{2}+n_{3}\right) / S\left(U\left(n_{1}\right) \times U\left(n_{2}\right) \times U\left(n_{3}\right)\right)
$$

is given in [1]. The existence is proved in [26] of at least one invariant Einstein metric for every three-locally-symmetric space. Furthermore in [23], the classification of invariant Einstein metrics is given for

$$
S p(l+m+n) / S p(l) \times S p(m) \times S p(m) \text { and } S O(l+m+n) / S O(l) \times S O(m) \times S O(m) .
$$

2010 Mathematics Subject Classification. Primary 53C25; Secondary 53C30, 17B20.

Key words and phrases. Einstein metric, three-locally-symmetric space, homogeneous space, involution. 
But the classification of three-locally-symmetric spaces is still incomplete, which leads to the incomplete classification of invariant Einstein metrics. In this paper, we complete the classification of three-locally-symmetric spaces for $G$ simple, and then classify invariant Einstein metrics.

The paper is organized as follows. In Section 2, we give the correspondence between the classification of three-locally-symmetric spaces $G / H$ and that of certain involution pairs of $G$. In Section 3, the classification of three-locally-symmetric spaces $G / H$ is given for $G$ simple based on the theory on involutions of compact Lie groups. We list them in Table 1 in Theorem 3.16 . Furthermore we prove the isotropy summands are pairwise nonisomorphic for the new threelocally-symmetric spaces. It makes the method of [23] valid to give the classification of invariant Einstein metrics on these three-locally-symmetric spaces in Section 4.

Remark 1.1. The minute that we uploaded this paper on www.arXiv.org, we received an Email from Prof. Yurii Nikonorov with the paper [27] on three-locally-symmetric spaces which were called generalized Wallach spaces. The classification of three-locally-symmetric spaces was obtained in [27] based on the classification of $\mathbb{Z}_{2} \times \mathbb{Z}_{2}$-symmetric spaces [5, 18, 21].

\section{Three-Locally-Symmetric spaces $G / H$ And involution pairs of $G$}

Assume that $G / H$ is a three-locally-symmetric space. Then

$$
\mathfrak{g}=\mathfrak{h} \oplus \mathfrak{p}_{1} \oplus \mathfrak{p}_{2} \oplus \mathfrak{p}_{3},
$$

and it is easy to see that the Lie brackets satisfy

$$
\left[\mathfrak{h}, \mathfrak{p}_{i}\right] \subset \mathfrak{p}_{i}, \quad\left[\mathfrak{p}_{i}, \mathfrak{p}_{i}\right] \subset \mathfrak{h}, \quad\left[\mathfrak{p}_{i}, \mathfrak{p}_{j}\right] \subset \mathfrak{p}_{k}
$$

for any $i \in\{1,2,3\}$ and $\{i, j, k\}=\{1,2,3\}$. Define a linear map $\theta_{1}$ on $\mathfrak{g}$ by

$$
\left.\theta_{1}\right|_{\mathfrak{h} \oplus \mathfrak{p}_{1}}=i d,\left.\quad \theta_{1}\right|_{\mathfrak{p}_{2} \oplus \mathfrak{p}_{3}}=-i d .
$$

By the equation (2.1), it is easy to check that

$$
\theta_{1}[X, Y]=\left[\theta_{1}(X), \theta_{1}(Y)\right] \text {, for any } X, Y \in \mathfrak{g} .
$$

It follows that $\theta_{1}$ is an automorphism, and then an involution of $\mathfrak{g}$. Similarly, define another linear map $\theta_{2}$ on $\mathfrak{g}$ by

$$
\left.\theta_{2}\right|_{\mathfrak{h} \oplus \mathfrak{p}_{2}}=i d,\left.\quad \theta_{2}\right|_{\mathfrak{p}_{1} \oplus \mathfrak{p}_{3}}=-i d,
$$

which is also an involution of $\mathfrak{g}$. Moreover we have $\theta_{1} \theta_{2}=\theta_{2} \theta_{1}, \mathfrak{h}=\left\{X \in \mathfrak{g} \mid \theta_{1}(X)=X, \theta_{2}(X)=\right.$ $X\}, \mathfrak{p}_{1}=\left\{X \in \mathfrak{g} \mid \theta_{1}(X)=X, \theta_{2}(X)=-X\right\}, \mathfrak{p}_{2}=\left\{X \in \mathfrak{g} \mid \theta_{1}(X)=-X, \theta_{2}(X)=X\right\}$, and $\mathfrak{p}_{3}=\left\{X \in \mathfrak{g} \mid \theta_{1}(X)=-X, \theta_{2}(X)=-X\right\}$.

On the other hand, let $G$ be a compact semisimple connected Lie group with the Lie algebra $\mathfrak{g}$ and $\rho, \varphi$ be involutions of $\mathfrak{g}$ satisfying $\rho \varphi=\varphi \rho$. Then we have a decomposition of $\mathfrak{g}$

$$
\mathfrak{g}=\mathfrak{h} \oplus \mathfrak{p}_{1} \oplus \mathfrak{p}_{2} \oplus \mathfrak{p}_{3},
$$

corresponding to $\rho, \varphi$, where $\mathfrak{h}=\{X \in \mathfrak{g} \mid \rho(X)=X, \varphi(X)=X\}, \mathfrak{p}_{1}=\{X \in \mathfrak{g} \mid \rho(X)=$ $X, \varphi(X)=-X\}, \mathfrak{p}_{2}=\{X \in \mathfrak{g} \mid \rho(X)=-X, \varphi(X)=X\}$, and $\mathfrak{p}_{3}=\{X \in \mathfrak{g} \mid \rho(X)=-X, \varphi(X)=$ $-X\}$. It is easy to check that

$$
\left[\mathfrak{h}, \mathfrak{p}_{i}\right] \subset \mathfrak{p}_{i}, \quad\left[\mathfrak{p}_{i}, \mathfrak{p}_{i}\right] \subset \mathfrak{h}, \quad\left[\mathfrak{p}_{i}, \mathfrak{p}_{j}\right] \subset \mathfrak{p}_{k}
$$

for any $i \in\{1,2,3\}$ and $\{i, j, k\}=\{1,2,3\}$. Let $H$ denote the connected Lie subgroup of $G$ with the Lie algebra $\mathfrak{h}$. If every $\mathfrak{p}_{i}$ for $i \in\{1,2,3\}$ is an irreducible adh-module, then $G / H$ is a three-locally-symmetric space.

In summary, there is a one-to-one correspondence between the set of three-locally-symmetric spaces and the set of commutative involution pairs of $\mathfrak{g}$ such that every $\mathfrak{p}_{i}$ for $i \in\{1,2,3\}$ is an irreducible adh-module. 


\section{THE CLASSIFICATION OF THREE-LOCALLY-SYMMETRIC SPACES}

The section is to give the classification of three-locally-symmetric spaces for a compact simple Lie group. By the discussion in Section 2, it turns to the classification of certain commutative involution pairs.

Let $G$ be a compact simple connected Lie group with the Lie algebra $\mathfrak{g}$ and $(\theta, \tau)$ be an involution pair of $G$ with $\theta \tau=\tau \theta$. Then for $\theta$, we have a decomposition,

$$
\mathfrak{g}=\mathfrak{k}+\mathfrak{m},
$$

where $\mathfrak{k}=\{X \in \mathfrak{g} \mid \theta(X)=X\}$ and $\mathfrak{m}=\{X \in \mathfrak{g} \mid \theta(X)=-X\}$. Since $\theta \tau=\tau \theta$, we have that

$$
\tau(X) \in \mathfrak{k} \text { for any } X \in \mathfrak{k},
$$

which implies that $\left.\tau\right|_{\mathfrak{k}}$ is an involution of $\mathfrak{k}$. Roughly to say, we can give the classification of commutative involution pairs of $\mathfrak{g}$ by studying the extension of an involution of $\mathfrak{k}$ to $\mathfrak{g}$. But an important problem is when an involution of $\mathfrak{k}$ can be extended to an involution of $\mathfrak{g}$.

Cartan and Gantmacher made great attributions on the classification of involutions on compact Lie groups. The theory on the extension of involutions of $\mathfrak{k}$ to $\mathfrak{g}$ can be found in [6], which is different in the method from that in [35. There are also some related discussion in [11, 12, 13. The following are the theories without proof.

Let $\mathfrak{t}_{1}$ be a Cartan subalgebra of $\mathfrak{k}$ and let $\mathfrak{t}$ be a Cartan subalgebra of $\mathfrak{g}$ containing $\mathfrak{t}_{1}$.

Theorem 3.1 (Gantmacher Theorem). Let the notations be as above. Then $\theta$ is conjugate with $\theta_{0} e^{\operatorname{ad} H}$ under Autg, where $\theta_{0}$ is a canonique involution and $H \in \mathfrak{t}_{1}$.

Let $\Pi=\left\{\alpha_{1}, \cdots, \alpha_{n}\right\}$ be a fundamental system of $\mathfrak{t}$ and $\phi=\sum_{i=1}^{n} m_{i} \alpha_{i}$ be the maximal root respectively. Let $\alpha_{i}^{\prime}=\frac{1}{2}\left(\alpha_{i}+\theta_{0}\left(\alpha_{i}\right)\right)$. Then $\Pi^{\prime}=\left\{\alpha_{1}^{\prime}, \cdots, \alpha_{l}^{\prime}\right\}$ consisting different elements in $\left\{\alpha_{1}^{\prime}, \cdots, \alpha_{n}^{\prime}\right\}$ is a fundamental system of $\mathfrak{g}_{0}$, where $\mathfrak{g}_{0}=\left\{X \in \mathfrak{g} \mid \theta_{0}(X)=X\right\}$. Denote by $\phi^{\prime}=\sum_{i=1}^{l} m_{i}^{\prime} \alpha_{i}^{\prime}$ the maximal root of $\mathfrak{g}_{0}$ respectively. Furthermore we have

Theorem 3.2 ([35]). If $H \neq 0$, then for some $i$, we can take $H$ satisfying

$$
\alpha_{i}^{\prime}=\alpha_{i} ; \quad\left\langle H, \alpha_{i}^{\prime}\right\rangle=\pi \sqrt{-1} ; \quad\left\langle H, \alpha_{j}^{\prime}\right\rangle=0, \forall j \neq i .
$$

Here $m_{i}^{\prime}=1$ or $m_{i}^{\prime}=2$.

Moreover, $\mathfrak{k}$ is described as follows.

Theorem 3.3 ([35). Let the notations be as above. Assume that $\alpha_{i}$ satisfies the identity (3.1).

(1) If $\theta_{0}=$ Id and $m_{i}=1$, then $\Pi-\left\{\alpha_{i}\right\}$ is the fundamental system of $\mathfrak{k}$, and $\phi$ and $-\alpha_{i}$ are the highest weights of $\operatorname{ad}_{\mathfrak{m}} \mathfrak{C}$.

(2) If $\theta_{0}=$ Id and $m_{i}=2$, then $\Pi-\left\{\alpha_{i}\right\} \cup\{-\phi\}$ is the fundamental system of $\mathfrak{k}$, and $-\alpha_{i}$ is the highest weight of $\operatorname{ad}_{\mathfrak{m}} \mathbb{C}^{k}$.

(3) If $\theta_{0} \neq I d$, then $\Pi^{\prime}-\left\{\alpha_{i}^{\prime}\right\} \cup\left\{\beta_{0}\right\}$ is the fundamental system of $\mathfrak{k}$, and $-\alpha_{i}$ is the highest weight of $\operatorname{ad}_{\mathfrak{m}} \mathbb{C}^{k}$.

Remark 3.4. In Theorem 3.3, the dimension of $C(\mathfrak{k})$, i.e., the center of $\mathfrak{k}$, is 1 for case (1); 0 for cases (2) and (3), $\beta_{0}$ in case (3) is the highest weight of $\operatorname{ad}_{\mathfrak{m}^{\mathbb{C}}} \mathfrak{k}$ for $\theta=\theta_{0}$ corresponding to $\Pi^{\prime}$.

Now for any involution $\tau^{\mathfrak{k}}$ of $\mathfrak{k}$, we can write

$$
\tau^{\mathfrak{k}}=\tau_{0}^{\mathfrak{k}} e^{\operatorname{ad} H^{\mathfrak{k}}},
$$

where $\tau_{0}^{\mathfrak{k}}$ is a canonique involution on $\mathfrak{k}, H^{\mathfrak{k}} \in \mathfrak{t}_{1}$ and $\tau_{0}^{\mathfrak{k}}\left(H^{\mathfrak{k}}\right)=H^{\mathfrak{k}}$. Since $e^{\operatorname{ad} H^{\mathfrak{k}}}$ is an innerautomorphism, naturally we can extend $e^{\text {add } H^{\mathfrak{k}}}$ to be an automorphism of $\mathfrak{g}$. Moreover,

Theorem 3.5 ([35]). The canonique involution $\tau_{0}^{\mathfrak{k}}$ can be extended to be an automorphism of $\mathfrak{g}$ if and only if $\tau_{0}^{\mathfrak{k}}$ keeps the weight system of $\mathrm{ad}_{\mathfrak{m}^{\mathfrak{c}}}$ invariant. 
If $C(\mathfrak{k}) \neq 0$, then $\operatorname{dim} C(\mathfrak{k})=1$. Thus $\tau_{0}^{\mathfrak{k}}(Z)=Z$ or $\tau_{0}^{\mathfrak{k}}(Z)=-Z$ for any $Z \in C(\mathfrak{k})$.

Theorem $3.6([35])$. Assume that $C(\mathfrak{k}) \neq 0$ and $\tau_{0}^{\mathfrak{k}}(Z)=Z$ for any $Z \in C(\mathfrak{k})$. If $\tau^{\mathfrak{k}}$ can be extended to be an automorphism of $\mathfrak{g}$, then $\tau^{\mathfrak{k}}$ can be extended to be an involution of $\mathfrak{g}$.

For the other cases, we have the following theorems.

Theorem $3.7([35])$. Assume that $C(\mathfrak{k})=0$, or $C(\mathfrak{k}) \neq 0$ but $\tau_{0}^{\mathfrak{k}}(Z)=-Z$ for any $Z \in C(\mathfrak{k})$. If $\tau$ is an automorphism of $\mathfrak{g}$ extended by an involution $\tau^{\mathfrak{k}}$ of $\mathfrak{k}$, then $\tau^{2}=I d$ or $\tau^{2}=\theta$. Furthermore, the following conditions are equivalent:

(1) There exists an automorphism $\tau$ of $\mathfrak{g}$ extended by $\tau^{\mathfrak{k}}$ which is an involution.

(2) Every automorphism $\tau$ of $\mathfrak{g}$ extended by $\tau^{\mathfrak{k}}$ is an involution.

Then it is enough to determine when the automorphism extended by $\tau^{\mathfrak{k}}$ is an involution.

Theorem 3.8 ([35]). Let $\tau_{0}$ be the automorphism of $\mathfrak{g}$ extended by the canonique involution $\tau_{0}^{\mathfrak{k}}$ on $\mathfrak{k}$. Then $\tau_{0}^{2}=I d$ except $\mathfrak{g}=A_{n}^{i}$ and $n$ is even. For $e^{\operatorname{ad} H^{\mathfrak{k}}}$, we have:

(1) If $\theta_{0} \neq I d$, then the natural extension of $e^{\operatorname{ad} H^{\mathfrak{k}}}$ is an involution.

(2) Assume that $\theta_{0}=I d$. Let $\alpha_{i_{1}}^{\prime}, \cdots, \alpha_{i_{k}}^{\prime}$ be the roots satisfying $\left\langle\alpha_{j}^{\prime}, H\right\rangle \neq 0$. Then the natural extension of $e^{\mathrm{ad} H^{\mathfrak{k}}}$ is an involution if and only if $\sum_{j=i_{1}}^{i_{k}} m_{j}^{\prime}$ is even.

In particular, for every cases in Theorem 3.3 ,

Theorem 3.9 ([6, 35]). If $\tau$ is an involution of $\mathfrak{g}$ extended by an involution $\tau^{\mathfrak{k}}$ on $\mathfrak{k}$, then every extension of $\tau^{\mathfrak{k}}$ is an involution of $\mathfrak{k}$, which is equivalent with $\tau$ or $\tau \theta$.

Up to now, we can obtain the classification of commutative involution pairs by that of $\left(\theta, \tau^{\mathfrak{k}}\right)$ based on the above theory. By Theorem 3.9, for an involution $\tau^{\mathfrak{k}}$ on $\mathfrak{k}$ which can be extended to an involution $\tau$ of $\mathfrak{g}$, we have two involution pairs $(\theta, \tau)$ and $(\theta, \theta \tau)$ which determine the same threelocally-symmetric space. So, without loss of generality, we denote by $\tau$ the natural extension of $\tau^{\mathfrak{k}}$. Let $\mathfrak{h}=\left\{X \in \mathfrak{k} \mid \tau^{\mathfrak{k}}(X)=X\right\}, \mathfrak{p}_{1}=\{X \in \mathfrak{k} \mid \tau(X)=-X\}, \mathfrak{p}_{2}=\{X \in \mathfrak{m} \mid \tau(X)=X\}$, and $\mathfrak{p}_{3}=\{X \in \mathfrak{m} \mid \tau(X)=-X\}$. We shall pick up certain pairs $\left(\theta, \tau^{\mathfrak{k}}\right)$ by the following steps.

Step 1: we obtain the classification of the pair $\left(\theta, \tau^{\mathfrak{k}}\right)$ satisfying that the extension $\tau$ of $\tau^{\mathfrak{k}}$ is an involution and $\mathfrak{p}_{1}, \mathfrak{p}_{2}, \mathfrak{p}_{3}$ are irreducible as adh-modules.

Step 2: among the pairs given by Step 1, we remain only one if several pairs determine the same three-locally-symmetric space.

By the above steps, we obtain the list of the following pairs $\left(\theta, \tau^{\mathfrak{k}}\right)$. Furthermore, we make some remarks on them.

Case $G=A_{l}(l \geq 1)$. The Dynkin diagram with the maximal root is

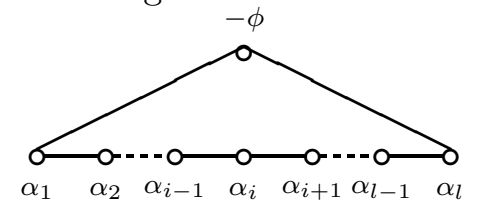

$(\operatorname{InP} 1) l=1, \theta=e^{\operatorname{ad} H}$, where $\left\langle H, \alpha_{1}\right\rangle=\pi \sqrt{-1} ;\left.\tau^{\mathfrak{k}}\right|_{C(\mathfrak{k})}=-I d$.

(InP2) $l \geq 3$ is odd, $\theta=e^{\operatorname{ad} H}$, where $\left\langle H, \alpha_{\frac{l+1}{2}}\right\rangle=\pi \sqrt{-1}$ and $\left\langle H, \alpha_{k}\right\rangle=0$ for any $\alpha_{k} \in$ $\Pi-\left\{\alpha_{i}\right\} ; \tau^{\mathfrak{k}}\left(\alpha_{k}\right)=\alpha_{l+1-k}$ for $k \neq \frac{l+1}{2}$ and $\left.\tau^{\mathfrak{k}}\right|_{C(\mathfrak{k})}=I d$.

(InP3) $l \geq 2, \theta=e^{\text {ad } H}$, where $\left\langle H, \alpha_{i}\right\rangle=\pi \sqrt{-1}$ for some $1 \leq i \leq\left[\frac{l+1}{2}\right]$ and $\left\langle H, \alpha_{k}\right\rangle=0$ for any $\alpha_{k} \in \Pi-\left\{\alpha_{i}\right\} ; \tau^{\mathfrak{k}}=e^{\text {ad } H_{1}}$, where $\left\langle H_{1}, \alpha_{j}\right\rangle=\pi \sqrt{-1}$ for some $\alpha_{j} \in \Pi-\left\{\alpha_{i}\right\}$ and $\left\langle H, \alpha_{k}\right\rangle=0$ for any $\alpha_{k} \in \Pi-\left\{\alpha_{i}, \alpha_{j}\right\}$. Furthermore, we may require that $1 \leq i \leq\left[\frac{l+1}{3}\right]$ and $2 i \leq j \leq\left[\frac{l+i+1}{2}\right]$ for Step 2.

Case $G=B_{l}(l \geq 2)$. The Dynkin diagram with the maximal root is 


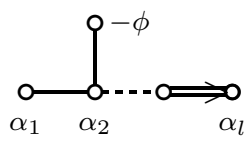

(InP4) $\theta=e^{\operatorname{ad} H}$, where $\left\langle H, \alpha_{i}\right\rangle=\pi \sqrt{-1}$ for some $2 \leq i \leq l$ and $\left\langle H, \alpha_{k}\right\rangle=0$ for any $\alpha_{k} \in \Pi-\left\{\alpha_{i}\right\} ; \tau^{\mathfrak{k}}=e^{\text {ad } H_{1}}$, where $\left\langle H_{1}, \alpha_{j}\right\rangle=\pi \sqrt{-1}$ for some $2 \leq j<i$ and $\left\langle H, \alpha_{k}\right\rangle=0$ for any $\alpha_{k} \in \Pi-\left\{\alpha_{i}, \alpha_{j}\right\} \cup\{-\phi\}$. Moreover, we may assume that $2<i \leq l$ and $\frac{i}{2} \leq j \leq i-1$.

(InP5) $\theta=e^{\operatorname{ad} H}$, where $\left\langle H, \alpha_{i}\right\rangle=\pi \sqrt{-1}$ for some $2 \leq i \leq l$ and $\left\langle H, \alpha_{k}\right\rangle=0$ for any $\alpha_{k} \in \Pi-\left\{\alpha_{i}\right\} ; \tau^{\mathfrak{k}}\left(\alpha_{1}\right)=-\phi, \tau^{\mathfrak{k}}(-\phi)=\alpha_{1}$, and $\tau^{\mathfrak{k}}\left(\alpha_{k}\right)=\alpha_{k}$ for any $\alpha_{k} \in \Pi-\left\{\alpha_{1}, \alpha_{i}\right\}$. Moreover, we may assume that $\frac{l+1}{2} \leq i \leq l$.

(InP6) $\theta=e^{\operatorname{ad} H}$, where $\left\langle H, \alpha_{i}\right\rangle=\pi \sqrt{-1}$ for some $2 \leq i \leq l$ and $\left\langle H, \alpha_{k}\right\rangle=0$ for any $\alpha_{k} \in \Pi-\left\{\alpha_{i}\right\} ; \tau^{\mathfrak{k}}=\tau_{0}^{\mathfrak{k}} e^{\text {ad } H_{1}}$, where $\tau_{0}^{\mathfrak{k}}\left(\alpha_{1}\right)=-\phi, \tau_{0}^{\mathfrak{k}}(-\phi)=\alpha_{1}$, and $\tau_{0}^{\mathfrak{k}}\left(\alpha_{k}\right)=\alpha_{k}$ for any $\alpha_{k} \in \Pi-\left\{\alpha_{1}, \alpha_{i}\right\},\left\langle H_{1}, \alpha_{j}\right\rangle=\pi \sqrt{-1}$ for some $2 \leq j<i$ and $\left\langle H, \alpha_{k}\right\rangle=0$ for any $\alpha_{k} \in$ $\Pi-\left\{\alpha_{i}, \alpha_{j}\right\} \cup\{-\phi\}$. Furthermore, we may require that $\left[\frac{2 l+3}{3}\right] \leq i \leq l$ and $\left[\frac{i+2}{2}\right] \leq j \leq 2 i-l$.

Case $G=C_{l}(l \geq 3)$. The Dynkin diagram with the maximal root is

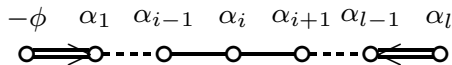

(InP7) $\theta=e^{\operatorname{ad} H}$, where $\left\langle H, \alpha_{i}\right\rangle=\pi \sqrt{-1}$ for some $1 \leq i \leq\left[\frac{l}{2}\right]$ and $\left\langle H, \alpha_{k}\right\rangle=0$ for any $\alpha_{k} \in \Pi-\left\{\alpha_{i}\right\} ; \tau^{\mathfrak{k}}=e^{\text {ad } H_{1}}$, where $\left\langle H_{1}, \alpha_{j}\right\rangle=\pi \sqrt{-1}$ for some $\alpha_{j} \in \Pi-\left\{\alpha_{i}, \alpha_{l},-\phi\right\}$ and $\left\langle H, \alpha_{k}\right\rangle=0$ for any $\alpha_{k} \in \Pi-\left\{\alpha_{i}, \alpha_{j}\right\} \cup\{-\phi\}$. Furthermore, we may require that $1 \leq i \leq\left[\frac{l}{3}\right]$ and $2 i \leq j \leq\left[\frac{l+i}{2}\right]$.

Case $G=D_{l}(l \geq 4)$. The Dynkin diagram with the maximal root is

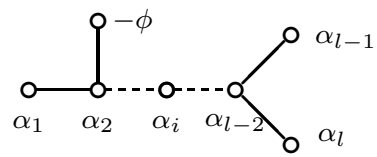

(InP8) $\theta=e^{\operatorname{ad} H}$, where $\left\langle H, \alpha_{i}\right\rangle=\pi \sqrt{-1}$ for some $2 \leq i \leq\left[\frac{l}{2}\right]$ and $\left\langle H, \alpha_{k}\right\rangle=0$ for any $\alpha_{k} \in \Pi-\left\{\alpha_{i}\right\} ; \tau^{\mathfrak{k}}=e^{\text {ad } H_{1}}$, where $\left\langle H_{1}, \alpha_{j}\right\rangle=\pi \sqrt{-1}$ for some $\alpha_{j} \in \Pi-\left\{\alpha_{1}, \alpha_{i}, \alpha_{l-1}, \alpha_{l}\right\}$ and $\left\langle H, \alpha_{k}\right\rangle=0$ for any $\alpha_{k} \in \Pi-\left\{\alpha_{i}, \alpha_{j}\right\} \cup\{-\phi\}$. Furthermore, we may require that $1 \leq i \leq\left[\frac{l}{3}\right]$ and $2 i \leq j \leq\left[\frac{l+i}{2}\right]$

(InP9) $\theta=e^{\operatorname{ad} H}$, where $\left\langle H, \alpha_{i}\right\rangle=\pi \sqrt{-1}$ for some $1 \leq i \leq l-2$ and $\left\langle H, \alpha_{k}\right\rangle=0$ for any $\alpha_{k} \in \Pi-\left\{\alpha_{i}\right\} ; \tau^{\mathfrak{k}}\left(\alpha_{l}\right)=\alpha_{l-1}$ and $\tau^{\mathfrak{k}}\left(\alpha_{l-1}\right)=\alpha_{l}$.

(InP10) $\theta=e^{\text {ad } H}$, where $\left\langle H, \alpha_{i}\right\rangle=\pi \sqrt{-1}$ for some $1 \leq i \leq l-2$ and $\left\langle H, \alpha_{k}\right\rangle=0$ for any $\alpha_{k} \in \Pi-\left\{\alpha_{i}\right\} ; \tau^{\mathfrak{k}}=\tau_{0}^{\mathfrak{k}} e^{\text {ad } H_{1}}$, where $\tau_{0}^{\mathfrak{k}}\left(\alpha_{l}\right)=\alpha_{l-1}$ and $\tau_{0}^{\mathfrak{k}}\left(\alpha_{l-1}\right)=\alpha_{l},\left\langle H_{1}, \alpha_{j}\right\rangle=\pi \sqrt{-1}$ for some $i<j \leq \frac{l+i-1}{2}$ and $\left\langle H, \alpha_{k}\right\rangle=0$ for any $\alpha_{k} \in \Pi-\left\{\alpha_{i}, \alpha_{j}\right\} \cup\{-\phi\}$.

$(\operatorname{InP} 11) \theta=e^{\operatorname{ad} H}$, where $\left\langle H, \alpha_{1}\right\rangle=\pi \sqrt{-1}$ and $\left\langle H, \alpha_{k}\right\rangle=0$ for any $\alpha_{k} \in \Pi-\left\{\alpha_{1}\right\} ;\left.\tau^{\mathfrak{k}}\right|_{C(\mathfrak{k})}=$ $-I d$.

(InP12) $\theta=e^{\operatorname{ad} H}$, where $\left\langle H, \alpha_{1}\right\rangle=\pi \sqrt{-1}$ and $\left\langle H, \alpha_{k}\right\rangle=0$ for any $\alpha_{k} \in \Pi-\left\{\alpha_{1}\right\} ; \tau^{\mathfrak{k}}=e^{\operatorname{ad} H_{1}}$, where $\left\langle H_{1}, \alpha_{l}\right\rangle=\pi \sqrt{-1}$ and $\left\langle H, \alpha_{k}\right\rangle=0$ for any $\alpha_{k} \in \Pi-\left\{\alpha_{1}, \alpha_{l}\right\}$.

Case $G=E_{6}$. The Dynkin diagram with the maximal root is

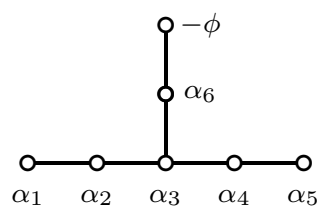


(InP13) $\theta=e^{\operatorname{ad} H}$, where $\left\langle H, \alpha_{1}\right\rangle=\pi \sqrt{-1}$ and $\left\langle H, \alpha_{k}\right\rangle=0$ for any $\alpha_{k} \in \Pi-\left\{\alpha_{1}\right\} ; \tau^{\mathfrak{k}}=e^{\operatorname{ad} H_{1}}$, where $\left\langle H_{1}, \alpha_{5}\right\rangle=\pi \sqrt{-1}$ and $\left\langle H, \alpha_{k}\right\rangle=0$ for any $\alpha_{k} \in \Pi-\left\{\alpha_{1}, \alpha_{5}\right\}$.

$(\operatorname{InP} 14) \theta=e^{\operatorname{ad} H}$, where $\left\langle H, \alpha_{6}\right\rangle=\pi \sqrt{-1}$ and $\left\langle H, \alpha_{k}\right\rangle=0$ for any $\alpha_{k} \in \Pi-\left\{\alpha_{6}\right\} ; \tau^{\mathfrak{k}}=e^{\operatorname{ad} H_{1}}$, where $\left\langle H_{1}, \alpha_{2}\right\rangle=\pi \sqrt{-1}$ and $\left\langle H, \alpha_{k}\right\rangle=0$ for any $\alpha_{k} \in \Pi-\left\{\alpha_{2}, \alpha_{6}\right\} \cup\{-\phi\}$.

$(I n P 15) \theta=e^{\operatorname{ad} H}$, where $\left\langle H, \alpha_{6}\right\rangle=\pi \sqrt{-1}$ and $\left\langle H, \alpha_{k}\right\rangle=0$ for any $\alpha_{k} \in \Pi-\left\{\alpha_{6}\right\} ; \tau^{\mathfrak{k}}\left(\alpha_{i}\right)=$ $\alpha_{6-i}$ for $i=1,2,3,4,5$.

Case $G=E_{7}$. The Dynkin diagram with the maximal root is

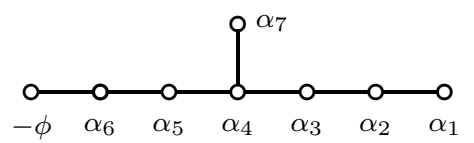

(InP16) $\theta=e^{\mathrm{ad} H}$, where $\left\langle H, \alpha_{6}\right\rangle=\pi \sqrt{-1}$ and $\left\langle H, \alpha_{k}\right\rangle=0$ for any $\alpha_{k} \in \Pi-\left\{\alpha_{6}\right\} ; \tau^{\mathfrak{k}}=e^{\text {ad } H_{1}}$, where $\left\langle H_{1}, \alpha_{2}\right\rangle=\pi \sqrt{-1}$ and $\left\langle H, \alpha_{k}\right\rangle=0$ for any $\alpha_{k} \in \Pi-\left\{\alpha_{2}, \alpha_{6}\right\} \cup\{-\phi\}$.

(InP17) $\theta=e^{\operatorname{ad} H}$, where $\left\langle H, \alpha_{7}\right\rangle=\pi \sqrt{-1}$ and $\left\langle H, \alpha_{k}\right\rangle=0$ for any $\alpha_{k} \in \Pi-\left\{\alpha_{7}\right\} ; \tau^{\mathfrak{k}}=e^{\operatorname{ad} H_{1}}$, where $\left\langle H_{1}, \alpha_{2}\right\rangle=\pi \sqrt{-1}$ and $\left\langle H, \alpha_{k}\right\rangle=0$ for any $\alpha_{k} \in \Pi-\left\{\alpha_{2}, \alpha_{7}\right\} \cup\{-\phi\}$.

(InP18) $\theta=e^{\operatorname{ad} H}$, where $\left\langle H, \alpha_{7}\right\rangle=\pi \sqrt{-1}$ and $\left\langle H, \alpha_{k}\right\rangle=0$ for any $\alpha_{k} \in \Pi-\left\{\alpha_{7}\right\} ; \tau^{\mathfrak{k}}=$ $\tau_{0}^{\mathfrak{k}} e^{\operatorname{ad} H_{1}}$, where $\tau_{0}^{\mathfrak{k}}\left(\alpha_{i}\right)=\alpha_{8-i}$ for $i=2,3,4,5,6, \tau_{0}^{\mathfrak{k}}\left(\alpha_{1}\right)=-\phi$, and $\tau_{0}^{\mathfrak{k}}(-\phi)=\alpha_{1},\left\langle H_{1}, \alpha_{4}\right\rangle=$ $\pi \sqrt{-1}$ and $i<j \leq l-3$ and $\left\langle H_{1}, \alpha_{k}\right\rangle=0$ for any $\alpha_{k} \in \Pi-\left\{\alpha_{4}, \alpha_{7}\right\} \cup\{-\phi\}$.

Case $G=E_{8}$. The Dynkin diagram with the maximal root is

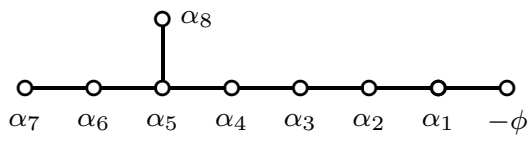

$(\operatorname{InP19}) \theta=e^{\operatorname{ad} H}$, where $\left\langle H, \alpha_{7}\right\rangle=\pi \sqrt{-1}$ and $\left\langle H, \alpha_{k}\right\rangle=0$ for any $\alpha_{k} \in \Pi-\left\{\alpha_{7}\right\} ; \tau^{\mathfrak{k}}=e^{\operatorname{ad} H_{1}}$, where $\left\langle H_{1}, \alpha_{1}\right\rangle=\pi \sqrt{-1}$ and $\left\langle H, \alpha_{k}\right\rangle=0$ for any $\alpha_{k} \in \Pi-\left\{\alpha_{1}, \alpha_{7}\right\} \cup\{-\phi\}$.

(InP20) $\theta=e^{\operatorname{ad} H}$, where $\left\langle H, \alpha_{7}\right\rangle=\pi \sqrt{-1}$ and $\left\langle H, \alpha_{k}\right\rangle=0$ for any $\alpha_{k} \in \Pi-\left\{\alpha_{7}\right\} ; \tau^{\mathfrak{k}}=e^{\operatorname{ad} H_{1}}$, where $\left\langle H_{1}, \alpha_{3}\right\rangle=\pi \sqrt{-1}$ and $\left\langle H, \alpha_{k}\right\rangle=0$ for any $\alpha_{k} \in \Pi-\left\{\alpha_{3}, \alpha_{7}\right\} \cup\{-\phi\}$.

Case $G=F_{4}$. The Dynkin diagram with the maximal root is

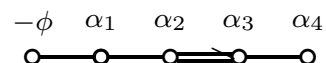

(InP21) $\theta=e^{\operatorname{ad} H}$, where $\left\langle H, \alpha_{4}\right\rangle=\pi \sqrt{-1}$ and $\left\langle H, \alpha_{k}\right\rangle=0$ for any $\alpha_{k} \in \Pi-\left\{\alpha_{4}\right\} ; \tau^{\mathfrak{k}}=e^{\operatorname{ad} H_{1}}$, where $\left\langle H_{1}, \alpha_{3}\right\rangle=\pi \sqrt{-1}$ and $\left\langle H, \alpha_{k}\right\rangle=0$ for any $\alpha_{k} \in \Pi-\left\{\alpha_{3}, \alpha_{4}\right\} \cup\{-\phi\}$.

(InP22) $\theta=e^{\operatorname{ad} H}$, where $\left\langle H, \alpha_{4}\right\rangle=\pi \sqrt{-1}$ and $\left\langle H, \alpha_{k}\right\rangle=0$ for any $\alpha_{k} \in \Pi-\left\{\alpha_{4}\right\} ; \tau^{\mathfrak{k}}=e^{\operatorname{ad} H_{1}}$, where $\left\langle H_{1}, \alpha_{1}\right\rangle=\pi \sqrt{-1}$ and $\left\langle H, \alpha_{k}\right\rangle=0$ for any $\alpha_{k} \in \Pi-\left\{\alpha_{1}, \alpha_{4}\right\} \cup\{-\phi\}$.

Remark 3.10. Consider $G=A_{l}$ when $l \geq 1$ is odd. We take the involution $\theta=e^{\text {ad } H}$, where $\left\langle H, \alpha_{\frac{l+1}{2}}\right\rangle=\pi \sqrt{-1}$ and $\left\langle H, \alpha_{k}\right\rangle=0$ for any $\alpha_{k} \in \Pi-\left\{\alpha_{i}\right\}$. Then $\tau^{\mathfrak{k}}$ defined by $\tau^{\mathfrak{k}}\left(\alpha_{k}\right)=\alpha_{\frac{l+1}{2}+k}$ and $\tau^{\mathfrak{k}}\left(\alpha_{\frac{l+1}{2}+k}\right)=\alpha_{k}$ for any $1 \leq k \leq \frac{l-1}{2}$ is an involution on $\mathfrak{k}$. By the above theory, if $\tau^{\mathfrak{k}}$ can be extended to an involution of $\mathfrak{g}$, we obtain $\tau^{\mathfrak{k}}\left(-\alpha_{\frac{l+1}{2}}\right)=\phi$. It is equivalent to $\left.\tau^{\mathfrak{k}}\right|_{C(\mathfrak{k})}=-I d$. If $l \geq 3, \mathfrak{p}_{1}$ is reducible. If $l=1$, we get the case $(\operatorname{In} \stackrel{2}{P} 1)$.

Remark 3.11. The case $(\operatorname{InP} 4)$ is valid for $i=1$ and $j$, which determines the same threelocally-symmetric space with $i^{\prime}=j$ and $j^{\prime}=j-1$.

Remark 3.12. If we take $\theta$ the case $(\operatorname{InP} 4)$ for $i=1$, then $\left.\tau^{\mathfrak{k}}\right|_{C(\mathfrak{k})}=-I d$ can be extended to an involution of $\mathfrak{g}$. This pair determines the same three-locally-symmetric space as the case (InP4) for $i=l$. 
Remark 3.13. The involution $\tau^{\mathfrak{k}}$ in cases $(\operatorname{InP} 5)$ and $(\operatorname{InP} 6)$ are outer-automorphism on $\mathfrak{k}$, which can be extended to an inner-automorphism of $\mathfrak{g}$.

Remark 3.14. Consider the following involution pair $(\theta, \tau)$ of $G=C_{l}$. First for the Dyinkin diagram of $C_{l}, \theta=e^{\text {ad } H}$ is an involution of $\mathfrak{g}$, where $\left\langle H, \alpha_{l}\right\rangle=\pi \sqrt{-1}$ and others zero. Then $\mathfrak{k}$ is the direct sum of $A_{l-1}$ and the center of one-dimension. Given an involution $\tau^{\mathfrak{k}}=e^{\operatorname{ad} H_{1}}$ of $\mathfrak{k}$, where $\left\langle H_{1}, \alpha_{j}\right\rangle=\pi \sqrt{-1}$ for some $j \in\{1,2, \cdots, l-1\}$ and $\left\langle H, \alpha_{k}\right\rangle=0$ for any $\alpha_{k} \in \Pi-\left\{\alpha_{j}, \alpha_{l}\right\}$. Then the extension of $\tau_{\mathfrak{k}}$ is an involution of $\mathfrak{g}$ and

(1) $\mathfrak{p}_{1}$ is an irreducible adh-module.

(2) By a result in [6], the extension of $\tau^{\mathfrak{k}}$ is $\tau$ or $\tau \theta$. Here $\tau$ is also denoted by $e^{\text {ad } H_{1}}$, where $\left\langle H_{1}, \alpha_{j}\right\rangle=\pi \sqrt{-1}$ and others zero.

We can prove that $\mathfrak{p}_{2}$ is a reducible adh-module. In fact, the Dynkin diagram of $\mathfrak{h}+\mathfrak{p}_{2}$, i.e. the set of fixed points of $\tau$, is

$$
\underset{-\phi}{\Longrightarrow} \alpha_{1} \alpha_{j-1} \quad \alpha_{j+1} \alpha_{l-1} \alpha_{l}
$$

Then by the definition of $\theta$, the Dynkin diagram of $\mathfrak{h}$ is

$$
\begin{array}{ccc}
\alpha_{1} \quad \alpha_{j-1} & \alpha_{j+1} \alpha_{l-1} \\
\text { ○---० } & 0-\cdots
\end{array}
$$

and on $\mathfrak{h}+\mathfrak{p}_{2},\left\langle H, \alpha_{l}\right\rangle=\pi \sqrt{-1},\langle H,-\phi\rangle=\pi \sqrt{-1}$, and others zero. That is, $\mathfrak{p}_{2}$ is a reducible adh-module.

Remark 3.15. This remark is in detail to describe $\mathfrak{p}_{1}, \mathfrak{p}_{2}, \mathfrak{p}_{3}$ as irreducible modules of adh for the case (InP21), which is given in [12]. For this case, $\mathfrak{p}_{1}, \mathfrak{p}_{2}, \mathfrak{p}_{3}$ are pairwise nonisomorphic. In fact, we can choose a fundamental system $\left\{\alpha_{1}, \alpha_{2}, \alpha_{3}, \alpha_{4}\right\}$ of $F_{4}$ such that the Dynkin diagram of $F_{4}$ corresponding to the fundamental system is

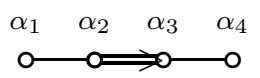

Consider the involution $\theta=e^{\text {ad } H}$ defined by

$$
\left\langle H, \alpha_{4}\right\rangle=\pi \sqrt{-1} ; \quad\left\langle H, \alpha_{j}\right\rangle=0, \forall j \neq 4 .
$$

Let $\phi=2 \alpha_{1}+3 \alpha_{2}+4 \alpha_{3}+2 \alpha_{4}$. Then the Dynkin diagram of $\mathfrak{k}$ is

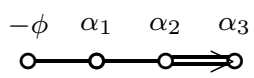

The involution $\tau$ is the extension of $\tau^{\mathfrak{k}}$, where $\tau^{\mathfrak{k}}=e^{\text {ad } H_{1}}$ satisfies

$$
\left\langle H_{1}, \alpha_{3}\right\rangle=\pi \sqrt{-1} ; \quad\left\langle H, \alpha_{1}\right\rangle=\left\langle H_{1}, \alpha_{2}\right\rangle=\left\langle H_{1},-\phi\right\rangle=0 .
$$

Then $\phi_{1}=-\left(\alpha_{2}+2 \alpha_{3}+2 \alpha_{4}\right)$ be the maximal root of $\mathfrak{k}$. Then the Dynkin diagram of $\mathfrak{h}$ is

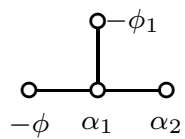

Let $\mathfrak{g}=\mathfrak{h} \oplus \mathfrak{p}_{1} \oplus \mathfrak{p}_{2} \oplus \mathfrak{p}_{3}$ be the decomposition of $\mathfrak{g}$ corresponding to $(\theta, \tau)$. Here $\mathfrak{k}=\mathfrak{h} \oplus \mathfrak{p}_{1}$, which is a decomposition of $\mathfrak{k}$ corresponding to the involution $\tau^{\mathfrak{k}}$. By Theorem 3.3. $\mathfrak{p}_{1}$ is the irreducible representation of $\mathfrak{h}$ with the highest weight $-\alpha_{3}$, which is a fundamental dominant weight corresponding to $\alpha_{2}$. Let $\mathfrak{k}^{1}=\{x \in \mathfrak{g} \mid \theta \tau(x)=x\}=\mathfrak{h} \oplus \mathfrak{p}_{3}$, which is a decomposition of $\mathfrak{k}^{1}$ corresponding to the involution $\left.\tau\right|_{\mathfrak{k}^{1}}$. The Dynkin diagram of $F_{4}$ corresponding to the fundamental system $\left\{\alpha_{1}, \alpha_{2}, \alpha_{3}^{\prime}=\alpha_{3}+\alpha_{4}, \alpha_{4}^{\prime}=-\alpha_{4}\right\}$ is

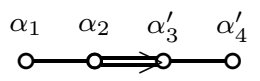


The involution $\theta \tau=e^{\operatorname{ad}\left(H+H_{1}\right)}$ satisfies

$$
\left\langle H+H_{1}, \alpha_{4}^{\prime}\right\rangle=\pi \sqrt{-1} ; \quad\left\langle H+H_{1}, \alpha_{1}\right\rangle=\left\langle H+H_{1}, \alpha_{2}\right\rangle=\left\langle H+H_{1}, \alpha_{3}^{\prime}\right\rangle=0 .
$$

Here the maximal root $2 \alpha_{1}+3 \alpha_{2}+4 \alpha_{3}^{\prime}+2 \alpha_{4}^{\prime}=2 \alpha_{1}+3 \alpha_{2}+4 \alpha_{3}+2 \alpha_{4}=\phi$. It follows that the Dynkin diagram of $\mathfrak{k}^{1}$ is

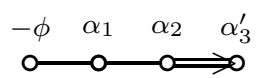

The involution $\tau=e^{\operatorname{ad} H_{1}}$ restricted on $\mathfrak{k}^{1}$ satisfies

$$
\left\langle H_{1}, \alpha_{3}^{\prime}\right\rangle=\pi \sqrt{-1} ; \quad\left\langle H, \alpha_{1}\right\rangle=\left\langle H_{1}, \alpha_{2}\right\rangle=\left\langle H_{1},-\phi\right\rangle=0 .
$$

Then $-\left(\alpha_{2}+2 \alpha_{3}\right)$ is the maximal root of $\mathfrak{k}$, and the Dynkin diagram of $\mathfrak{h}$ is

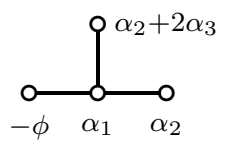

By Theorem 3.3, $\mathfrak{p}_{3}$ is the irreducible representation of $\mathfrak{h}$ with the highest weight $-\alpha_{3}^{\prime}$. For the fundamental system $\left\{-\phi, \alpha_{1}, \alpha_{2},-\phi_{1}\right\}, \mathfrak{p}_{3}$ is the irreducible representation of $\mathfrak{h}_{1}$ with the highest weight $-\left(\alpha_{1}+2 \alpha_{2}+3 \alpha_{3}+\alpha_{4}\right)$, which is a fundamental dominant weight corresponding to $-\phi$. The discussion for $\mathfrak{p}_{2}$ is similar. Finally, for the fundamental system $\left\{-\phi, \alpha_{1}, \alpha_{2},-\phi_{1}\right\}$ of $\mathfrak{h}$, we conclude that the highest weights of $\mathfrak{p}_{1}, \mathfrak{p}_{2}$ and $\mathfrak{p}_{3}$ as adh modules are fundamental dominant weights of $\mathfrak{h}$ corresponding to $\alpha_{2},-\phi_{1}$ and $-\phi$ respectively, which are pairwise nonisomorphic.

By the above theory, we classify three-locally-symmetric spaces as follows.

Theorem 3.16. The classification of three-locally-symmetric spaces $G / H$ for a connected, compact and simple Lie group $G$ is given in Table 1. In Table $1, A_{1}=B_{1}=C_{1}, B_{2}=C_{2}, A_{3}=D_{3}$, $D_{1}=T$ and $A_{0}=B_{0}=C_{0}=D_{0}=e$.

\begin{tabular}{|c|c|c|c|c|c|c|}
\hline Type & $G \quad\left(\theta, \tau^{\mathfrak{k}}\right)$ & $H$ & Type & $G$ & $\left(\theta, \tau^{\mathfrak{k}}\right)$ & $H$ \\
\hline$A-I$ & $(\operatorname{InP} 1)$ & $e$ & $A-I I$ & $A_{l}$ & $(\operatorname{InP2})$ & $\begin{array}{c}T \times A_{\frac{l-1}{2}} \\
l \geq 3 \text { is odd }\end{array}$ \\
\hline$\overline{A-I I I}$ & $A_{l} \quad(\operatorname{InP} 3)$ & $\begin{array}{c}T^{2} \times A_{i-1} \times A_{j-i-1} \times A_{l-j} \\
1 \leq i \leq\left[\frac{l+1}{3}\right] \\
2 i \leq j \leq\left[\frac{l+i+1}{2}\right]\end{array}$ & $B-I$ & $B_{l}$ & $(\operatorname{InP} 4)$ & $\begin{array}{c}B_{l-i} \times D_{j} \times D_{i-j} \\
2<i \leq l \\
\frac{i}{2} \leq j \leq i-1\end{array}$ \\
\hline$B-I I$ & (InP5) & $\begin{array}{c}B_{i-1} \times B_{l-i} \\
\frac{l+1}{2} \leq i \leq l\end{array}$ & $B-I I I$ & $B_{l}$ & $(\operatorname{InP} 6)$ & $\begin{array}{c}B_{i-1} \times B_{i-j} \times B_{l-i} \\
{\left[\frac{2 l+3}{3}\right] \leq i \leq l} \\
{\left[\frac{i+2}{2}\right] \leq j \leq 2 i-l}\end{array}$ \\
\hline$\overline{C-I}$ & $\begin{array}{ll}C_{l} & \left(\operatorname{In} P^{r}\right)\end{array}$ & $\begin{array}{c}C_{i} \times C_{j-i} \times C_{l-j} \\
1 \leq i \leq\left[\frac{l}{3}\right] \\
2 i \leq j \leq\left[\frac{l+i}{2}\right]\end{array}$ & $D-I$ & $\overline{D_{l}}$ & $(\operatorname{InP} 8)$ & $\begin{array}{c}D_{i} \times D_{j-i} \times D_{l-j} \\
1 \leq i \leq\left[\frac{l}{3}\right] \\
2 i \leq j \leq\left[\frac{l+i}{2}\right]\end{array}$ \\
\hline$\overline{D-I I}$ & $(\operatorname{InP9)}$ & $\begin{array}{l}B_{i-1} \times D_{l-i} \\
1 \leq i \leq l-2\end{array}$ & $D-I I I$ & $\overline{D_{l}}$ & $(\operatorname{InP10})$ & $\begin{array}{c}D_{i} \times B_{j-i} \times B_{l-j-1} \\
1 \leq i \leq l-2 \\
i \leq j \leq\left[\frac{l+i-1}{2}\right]\end{array}$ \\
\hline$D-I V$ & $D_{l} \quad(\operatorname{InP} 11)$ & $D_{l-1}$ & $D-V$ & $D_{l}$ & (InP12) & $T^{2} \times A_{l-2}$ \\
\hline$E_{6}-I$ & (InP13) & $T^{2} \times D_{4}$ & $E_{6}-I I$ & $E_{6}$ & $(\operatorname{InP} 14)$ & $T \times A_{1} \times A_{1} \times A_{3}$ \\
\hline$E_{6}-I I I$ & $E_{6} \quad(\operatorname{InP} 15)$ & $A_{1} \times C_{3}$ & $E_{7}-I$ & $E_{7}$ & $(\operatorname{InP16})$ & $A_{1} \times A_{1} \times A_{1} \times D_{4}$ \\
\hline$E_{7}-I I$ & $(\operatorname{InP17)}$ & $T \times A_{1} \times A_{5}$ & $E_{7}-I I I$ & $E_{7}$ & $(\operatorname{InP18)}$ & $D_{4}$ \\
\hline$E_{8}-I$ & $E_{8} \quad(\operatorname{InP} 19)$ & $A_{1} \times A_{1} \times D_{6}$ & $E_{8}-I I$ & $E_{8}$ & $(\operatorname{InP20)}$ & $D_{4} \times D_{4}$ \\
\hline$F_{4}-I$ & $\begin{array}{ll}F_{4} \quad(\operatorname{InP21}) \\
\end{array}$ & $D_{4}$ & $F_{4}-I I$ & $F_{4}$ & (InP22) & $A_{1} \times A_{1} \times C_{2}$ \\
\hline
\end{tabular}

Table 1: Classification of three-locally-symmetric spaces 
Remark 3.17. The well-known examples of three-locally-symmetric spaces are the following:

(1) $S U(2)=S U(2) /\{e\}$,

(2) $S U\left(n_{1}+n_{2}+n_{3}\right) / S\left(U\left(n_{1}\right) \times U\left(n_{2}\right) \times U\left(n_{3}\right)\right)$,

(3) $S O(l+m+n) / S O(l) \times S O(m) \times S O(n)$,

(4) $S O(2 n) / U(1) \times U(n-1)$,

(5) $S p(l+m+n) / S p(l) \times S p(m) \times S p(m)$,

(6) $E_{6} / U(1) \times U(1) \times \operatorname{Spin}(8)$,

(7) $F_{4} / \operatorname{Spin}(8)$.

The first one is the three-locally-symmetric space of type $A$-I in Table 1 of Theorem 3.16, the second one is of type $A$-III, the third one corresponds to types $B$-I, $B$-II, $B$-III, $D$-I, $D$-II, $D$-III, and $D$-IV, the fourth one is of type $D-\mathrm{V}$, the fifth one is of type $C$-I, the sixth one is of type $E_{6}$-I, and the seventh one is of type $F_{4}$-I. For the above cases, $\mathfrak{p}_{1}, \mathfrak{p}_{2}$ and $\mathfrak{p}_{3}$ have been proved to be irreducible, and pairwise nonisomorphic with respect to the adjoint action of the Lie algebra $\mathfrak{h}$ on $\mathfrak{p}$ except $S O(n+2) / S O(n)$, which is of type $B$-II for $i=l$ and $D$-IV.

By Theorem 3.16, we obtain in Table 2 the dimensions of $\mathfrak{p}_{1}, \mathfrak{p}_{2}$ and $\mathfrak{p}_{3}$ for the following cases. Clearly $\mathfrak{p}_{1}, \mathfrak{p}_{2}$ and $\mathfrak{p}_{3}$ are pairwise nonisomorphic for types $A$-II, $E_{6}$-III and $E_{7}$-II. We can prove

Table 2: The dimensions of $\mathfrak{p}_{1}, \mathfrak{p}_{2}$ and $\mathfrak{p}_{3}$

\begin{tabular}{cccc|cccc}
\hline Type & $\operatorname{dim} \mathfrak{p}_{1}$ & $\operatorname{dim} \mathfrak{p}_{2}$ & $\operatorname{dim} \mathfrak{p}_{3}$ & Type & $\operatorname{dim} \mathfrak{p}_{1}$ & $\operatorname{dim} \mathfrak{p}_{2}$ & $\operatorname{dim} \mathfrak{p}_{3}$ \\
\hline$A$-II & $\frac{(l-1)(l+3)}{4}$ & $\frac{(l+1)(l+3)}{4}$ & $\frac{(l-1)(l+1)}{4}$ & $E_{6}$-II & 16 & 16 & 24 \\
\hline$E_{6}$-III & 14 & 28 & 12 & $E_{7}$-I & 32 & 32 & 32 \\
\hline$E_{7}$-II & 24 & 30 & 40 & $E_{7}$-III & 35 & 35 & 35 \\
\hline$E_{8}$-I & 48 & 64 & 64 & $E_{8}$-II & 64 & 64 & 64 \\
\hline$F_{4}$-II & 20 & 8 & 8 & & & & \\
\hline
\end{tabular}

the same result for the other cases similar to Remark 3.15. In summary, we have the following theorem.

Theorem 3.18. Let $G / H$ be a three-locally-symmetric space in Theorem 3.16 with the decomposition $\mathfrak{p}=\mathfrak{p}_{1} \oplus \mathfrak{p}_{2} \oplus \mathfrak{p}_{3}$. Then $\mathfrak{p}_{1}, \mathfrak{p}_{2}, \mathfrak{p}_{3}$ are pairwise nonisomorphic with respect to the adjoint action of the Lie algebra $\mathfrak{h}$ on $\mathfrak{p}$ except types $B-I I$ for $i=l$ and $D-I V$.

\section{Einstein METRICS ON THREE-LOCALLY-SYMMETRIC SPACES}

There are some study on the geometry of three-locally-symmetric spaces. In particular, a lot of study on invariant Einstein metrics has been done for some three-locally-symmetric spaces independently. For example,

(1) The flag manifolds $S U(3) / T_{\max }, S p(3) / S p(1) \times S p(1) \times S p(1), F_{4} / S p i n(8)$ known as Wallach spaces admit invariant Riemannian metrics of positive section curvature ([33]). The invariant Einstein metrics on the first space are classified in [14, on the other two spaces in [30. In any case, there exactly four invariant Einstein metrics up to proportionality.

(2) The invariant Einstein metrics on the Kähler C-spaces $S U\left(n_{1}+n_{2}+n_{3}\right) / S\left(U\left(n_{1}\right) \times\right.$ $\left.U\left(n_{2}\right) \times U\left(n_{3}\right)\right), S O(2 n) / U(1) \times U(n-1), E_{6} / U(1) \times U(1) \times S p i n(8)$ are classified in [20]. Every spaces admits four invariant Einstein metrics up to proportionality. Another approach to $S U\left(n_{1}+n_{2}+n_{3}\right) / S\left(U\left(n_{1}\right) \times U\left(n_{2}\right) \times U\left(n_{3}\right)\right)$ is given in [1].

(3) The Lie group $S U(2)$ considered as $S U(2) /\{e\}$ admits only one left-invariant Einstein metric which is a metric of constant curvature [7].

(4) It is proved in [26] that every three-locally-symmetric space admits at least on invariant Einstein metric. Furthermore, it is proved in 23 that $S p(l+m+n) / S p(l) \times S p(m) \times S p(n)$ admits exactly four invariant Einstein metrics up to proportionality and that $S O(l+m+n) / S O(l) \times$ $S O(m) \times S O(n)$ admits one, two, three or four invariant Einstein metrics up to proportionality. 
In particular, it is demonstrated in [19] that $S O(n+2) / S O(n)$ admits just one Einstein metric up to isometry and homothety for $n \geq 3$, the space $S O(4) / S O(2)$ has two such metrics from the classification theorem for five dimensional homogeneous compact Einstein manifolds [2].

In summary, the classification of invariant Einstein metrics on three-locally-symmetric spaces are well-known for types $A$-I, $A$-III, $B$-I, $B$-II, $B$-III, $C$-I, $D$-I, $D$-II, $D$-III, $D$-IV $D$-V, $E_{6}$-I and $F_{4}$-I in Theorem 3.16 .

In the following, assume that $G / H$ is a three-locally-symmetric space in Theorem 3.16 except the above cases. By Theorem 3.18, in the decomposition $\mathfrak{p}=\mathfrak{p}_{1} \oplus \mathfrak{p}_{2} \oplus \mathfrak{p}_{3}, \mathfrak{p}_{1}, \mathfrak{p}_{2}, \mathfrak{p}_{3}$ are pairwise nonisomorphic with respect to the adjoint action of the Lie algebra $\mathfrak{h}$ on $\mathfrak{p}$. Then we can give the classification of invariant Einstein metrics on these spaces following the theory in [23, 26, 34.

Let $d_{i}$ denote the dimension of $\mathfrak{p}_{i}$, and let $\left\{e_{i}^{j}\right\}$ be an orthonormal basis in $\mathfrak{p}_{i}$ with respect to $\langle\cdot, \cdot\rangle=-B(\cdot, \cdot)$, where $i=1,2,3$ and $1 \leq j \leq d_{i}=\operatorname{dim} \mathfrak{p}_{i}$. Define the expression $\left[\begin{array}{c}k \\ i j\end{array}\right]$ by

$$
\left[\begin{array}{c}
k \\
i j
\end{array}\right]=\sum_{\alpha, \beta, \gamma}\left\langle\left[e_{i}^{\alpha}, e_{j}^{\beta}\right], e_{k}^{\gamma}\right\rangle^{2}
$$

where $\alpha, \beta, \gamma$ range from 1 to $d_{i}, d_{j}, d_{k}$ respectively. Then $\left[\begin{array}{c}k \\ i j\end{array}\right]$ are symmetric in all three indices and $\left[\begin{array}{c}k \\ i j\end{array}\right]=0$ if two indices concide. Let $c_{i}$ be the Casimir constant of the adjoint representation of $\mathfrak{h}$ on $\mathfrak{p}_{i}$. If $\left\{e_{0}^{j}\right\}_{1 \leq j \leq \operatorname{dim} \mathfrak{h}}$ is an orthonormal basis in $\mathfrak{h}$ with respect to $\langle\cdot, \cdot\rangle$ and $e$ is an arbitrary unit vector in $\mathfrak{p}_{i}$, then

$$
c_{i}=\sum_{j}\left\langle\left[e_{0}^{j}, e\right],\left[e_{0}^{j}, e\right]\right\rangle .
$$

For three-locally-symmetric spaces, by [23, 34],

$$
2 A=\left[\begin{array}{c}
k \\
i j
\end{array}\right]+\left[\begin{array}{c}
j \\
i k
\end{array}\right]=d_{i}\left(1-2 c_{i}\right)
$$

Let $\rho$ be an invariant metric on $G / H$. We identity it with the corresponding adh-invariant $(\cdot, \cdot)$ on $\mathfrak{p}$. Since $\mathfrak{p}_{i}$ are irreducible and pairwise nonisomorphic, we have

$$
(\cdot, \cdot)=\left.\left.\left.x_{1}\langle\cdot, \cdot\rangle\right|_{\mathfrak{p}_{1}} \oplus x_{2}\langle\cdot, \cdot\rangle\right|_{\mathfrak{p}_{2}} \oplus x_{3}\langle\cdot, \cdot\rangle\right|_{\mathfrak{p}_{3}}
$$

for some positive real numbers $x_{i}$. The Ricci curvature $\operatorname{Ric}(\cdot, \cdot)$ of the metric $(\cdot, \cdot)$ is also adhinvariant. It is easy to see

$$
\left.\operatorname{Ric}(\cdot, \cdot)\right|_{\mathfrak{p}_{i}}=\left.r_{i}(\cdot, \cdot)\right|_{\mathfrak{p}_{i}}
$$

for some real numbers $r_{i}$. As that given in [26], we have the following formula

$$
r_{i}=\frac{1}{2 x_{i}}+\frac{A}{2 d_{i}}\left(\frac{x_{i}}{x_{j} x_{k}}-\frac{x_{k}}{x_{i} x_{j}}-\frac{x_{j}}{x_{i} x_{k}}\right) .
$$

Here $\{i, j, k\}=\{1,2,3\}$. Put $a_{i}=\frac{A}{d_{i}}$. Then we have

$$
\left\{\begin{array}{l}
r_{1}=\frac{1}{2 x_{1}}+\frac{a_{1}}{2}\left(\frac{x_{1}}{x_{2} x_{3}}-\frac{x_{2}}{x_{1} x_{3}}-\frac{x_{3}}{x_{1} x_{2}}\right) \\
r_{2}=\frac{1}{2 x_{2}}+\frac{a_{2}}{2}\left(\frac{x_{2}}{x_{1} x_{3}}-\frac{x_{1}}{x_{2} x_{3}}-\frac{x_{3}}{x_{1} x_{2}}\right) \\
r_{3}=\frac{1}{2 x_{3}}+\frac{a_{3}}{2}\left(\frac{x_{3}}{x_{1} x_{2}}-\frac{x_{1}}{x_{2} x_{3}}-\frac{x_{2}}{x_{1} x_{3}}\right)
\end{array}\right.
$$


Now the invariant metric $(\cdot, \cdot)$ is Einstein if and only if $r_{1}=r_{2}=r_{3}$. If $a_{i}=a_{j}$ for $i \neq j$, then the equations $r_{i}=r_{j}$ and $r_{i}=r_{k}$ for $k \neq i, j$ become

$$
\left\{\begin{array}{l}
\left(x_{j}-x_{i}\right)\left(x_{k}-2 a_{i}\left(x_{i}+x_{j}\right)\right)=0, \\
x_{j}\left(x_{k}-x_{i}\right)+\left(a_{i}+a_{k}\right)\left(x_{i}^{2}-x_{k}^{2}\right)+\left(a_{k}-a_{i}\right) x_{j}^{2}=0
\end{array}\right.
$$

If $x_{j}=x_{i}$, then the second equation is

$$
\left(1-2 a_{k}\right) x_{i}^{2}-x_{i} x_{k}+\left(a_{i}+a_{k}\right) x_{k}^{2}=0 .
$$

If $a_{k}=1 / 2$, we have only one family of proportional Einstein metrics. Otherwise, $1-2 a_{k}>0$, hence, all real solutions of the equation (4.2) are positive. Then there exist one family of proportional Einstein metrics for $\Delta_{1}=0$, two families for $\Delta_{1}>0$, and none for $\Delta_{1}<0$. Here $\Delta_{1}$ is the discriminant of (4.2). If $x_{k}=2 a_{i}\left(x_{i}+x_{j}\right)$, then the second equation is

$$
\left(a_{i}+a_{k}\right)\left(1-4 a_{i}^{2}\right) x_{i}^{2}-\left(1-2 a_{i}+8 a_{i}^{2}\left(a_{i}+a_{k}\right)\right) x_{i} x_{j}+\left(a_{i}+a_{k}\right)\left(1-4 a_{i}^{2}\right) x_{j}^{2}=0 .
$$

If $a_{i}=1 / 2$ then the equation (4.3) has no solution. Otherwise, $1-4 a_{i}^{2}>0$, hence, all real roots of the equation (4.3) are positive. Then there exist one family of proportional Einstein metrics for the discriminant $\Delta_{2}=0$, two families for $\Delta_{2}>0$, and none for $\Delta_{2}<0$. Here $\Delta_{2}$ is the discriminant of (4.3).

In particular, if $a_{1}=a_{2}=a_{3}$, then we have the following theorem.

Theorem 4.1 ([23] Theorem 3). If $G / H$ is a three-locally-symmetric space in Theorem 3.16 satisfying $a_{1}=a_{2}=a_{3}$, then, for $a_{1} \notin\left\{\frac{1}{2}, \frac{1}{4}\right\}, G / H$ admits exactly four nonproportional invariant Einstein metrics. The parameters $\left\{x_{1}, x_{2}, x_{3}\right\}$ has the form $(t, t, t),\left(\left(1-2 a_{1}\right) t, 2 a_{1} t, 2 a_{1} t\right)$, $\left(2 a_{1} t,\left(1-2 a_{1}\right) t, 2 a_{1} t\right)$, or $\left(2 a_{1} t, 2 a_{1} t,\left(1-2 a_{1}\right) t\right)$. For $a_{1}=\frac{1}{2}$ and $a_{1}=\frac{1}{4}$, every invariant Einstein metric is proportional to the standard metric.

The following is the method for calculating $c_{i}$ given in 23. In detail, $\mathfrak{k}_{i}=\mathfrak{h} \oplus \mathfrak{p}_{i}$ is a subalgebra of $\mathfrak{g}$. Let $K_{i}$ be the connect Lie subgroup in $G$ with the Lie algebra $\mathfrak{k}_{i}$. In this case, the homogeneous spaces $K_{i} / H$ and $G / K_{i}$ are locally symmetric [7]. If $K_{i}$ does not act almost effectively on $M=K / H$, consider its subgroup acting on $M=K_{i} / H=\widetilde{K_{i}} / \widetilde{H}$ almost effectively, here $\widetilde{H}$ denotes the corresponding isotropy group. The pair of algebras $\left(\widetilde{\mathfrak{k}_{i}}, \widetilde{\mathfrak{h}}\right)$ is irreducible symmetric [7]. If $\widetilde{\mathfrak{k}_{i}}$ is simple, then its Killing form $B_{\widetilde{k_{i}}}$ is proportional to the restriction of the Killing form of $\mathfrak{g}$ to $\widetilde{\mathfrak{k}_{i}}$, i.e., $B_{\widetilde{k_{i}}}=\left.\gamma_{i} B\right|_{\widetilde{k_{i}}}$. By Lemma 1 in [23],

$$
c_{i}=\gamma_{i} / 2
$$

It follows that

$$
a_{i}=\frac{A}{d_{i}}=\frac{1-\gamma_{i}}{2}
$$

From the above formulae and results in [23, 26, 34], we can classify invariant Einstein metrics on three-locally-symmetric spaces case by case.

4.1. Invariant Einstein metrics on the three-locally-symmetric space of type $A$-II. For this case, $\mathfrak{h} \oplus \mathfrak{p}_{2}=C_{k}$, where $l=2 k-1$ for $k \geq 2$. By the table for $\gamma_{i} \geq \frac{1}{2}$ given in [15],

$$
\gamma_{2}=\frac{k+1}{2 k}
$$

In fact, there is a method to compute every $\gamma_{i}$ in [15]. Here for three-locally-symmetric spaces, from (4.1) and the dimensions in Table 2, we calculate directly

$$
\gamma_{1}=\frac{1}{2}, \quad \gamma_{3}=\frac{k-1}{2 k}
$$


It follows that

$$
a_{1}=\frac{1}{4}, \quad a_{2}=\frac{k-1}{4 k}, \quad a_{3}=\frac{k+1}{4 k} .
$$

Let $x_{1}=1$. The equations $r_{1}=r_{2}=r_{3}$ are equivalent to

$$
\left\{\begin{array}{l}
x_{2}^{2}-(2 k+1) x_{3}^{2}+4 k x_{2} x_{3}-4 k x_{2}+2 k+1=0, \\
x_{2}^{2}-x_{3}^{2}+2 x_{3}-2 x_{2}+\frac{1}{k}=0 .
\end{array}\right.
$$

Excluding the summand containing $x_{2}^{2}$ form the first equation, we obtain

$$
x_{2}\left(4 k x_{3}-4 k+2\right)=2 k x_{3}^{2}+2 x_{3}+\frac{1}{k}-2 k-1 .
$$

For this case, $4 k x_{3}-4 k+2 \neq 0$. Expressing $x_{2}$ by $x_{3}$ from (4.4) and inserting it into second one, we obtain

$$
\begin{aligned}
& 12 k^{4} x_{3}^{4}-\left(48 k^{4}-8 k^{3}\right) x_{3}^{3}+\left(72 k^{4}-36 k^{3}-4 k^{2}\right) x_{3}^{2} \\
& -\left(48 k^{4}-48 k^{3}+4 k^{2}+4 k\right) x_{3}+12 k^{4}-20 k^{3}+7 k^{2}+2 k-1=0 .
\end{aligned}
$$

Denote by $U_{0}\left(x_{3}\right)$ the left side of the above equation. Similar to [23], define Sturm's series by

$$
\begin{aligned}
& U_{1}\left(x_{3}\right)=48 k^{4} x_{3}^{3}-\left(144 k^{4}-24 k^{3}\right) x_{3}^{2}+\left(144 k^{4}-72 k^{3}-8 k^{2}\right) x_{3}-48 k^{4}+48 k^{3}-4 k^{2}-4 k, \\
& U_{2}\left(x_{3}\right)=\left(6 k^{3}+3 k^{2}\right) x_{3}^{2}-\left(12 k^{3}-2 k^{2}-\frac{8}{3} k\right) x_{3}+6 k^{3}-4 k^{2}-\frac{7}{6} k+\frac{5}{6}, \\
& U_{3}\left(x_{3}\right)=\frac{32 k}{27(2 k+1)^{2}}\left[\left(108 k^{4}-18 k^{3}-12 k^{2}+4 k\right) x_{3}-108 k^{4}+36 k^{3}+27 k^{2}-7 k-1\right], \\
& U_{4}\left(x_{3}\right)=-\frac{27\left(4 k^{2}-1\right)^{2}\left(27 k^{4}-9 k^{2}+1\right)}{4\left(-54 k^{3}+9 k^{2}+6 k-2\right)^{2}} .
\end{aligned}
$$

It follows that

$$
\begin{aligned}
& U_{0}(0)>0, U_{1}(0)<0, U_{2}(0)>0, U_{3}(0)<0, U_{4}(0)<0 \\
& U_{0}(\infty)>0, U_{1}(\infty)>0, U_{2}(\infty)>0, U_{3}(\infty)>0, U_{4}(\infty)<0 .
\end{aligned}
$$

Denote by $Z(0)$ the number of the sign changes in the series $U_{0}(0), U_{1}(0), U_{2}(0), U_{3}(0), U_{4}(0)$ (neglecting zeros) and $Z(\infty)$ the number of sign changes in $U_{0}(\infty), U_{1}(\infty), U_{2}(\infty), U_{3}(\infty)$, $U_{4}(\infty)$, where $U_{i}(\infty)$ denotes the leading coefficient of $U_{i}\left(x_{3}\right)$ which defines the sign of $U_{i}\left(x_{3}\right)$ as $x_{i} \rightarrow \infty$. By Sturm's theorem [32], the number of real roots of (4.1) on $(0, \infty)$ is equal to $Z(0)-Z(\infty)=2$ since $U_{0}(0) \neq 0$. By the discussion in [23], the homogeneous manifold of type $A$-II in Theorem 3.16 admits exactly two invariant Einstein metrics up to proportionality.

4.2. Invariant Einstein metrics on the three-locally-symmetric space of type $E_{6}$-II. For this case, $\mathfrak{h} \oplus \mathfrak{p}_{1}=A_{1} \oplus A_{5}$ and $\mathfrak{p}_{1} \subset A_{5}$. By the tables in [15], $\gamma_{1}=\frac{1}{2}$. From (4.1) and the dimensions in Table $2, \gamma_{2}=\frac{1}{2}$ and $\gamma_{3}=\frac{2}{3}$. It follows that

$$
a_{1}=a_{2}=\frac{1}{4}, \quad a_{3}=\frac{1}{6} .
$$

If $x_{1}=x_{2}$, then the equation (4.2) is

$$
\frac{2}{3} x_{1}^{2}-x_{1} x_{3}+\frac{5}{12} x_{3}^{2}=0 .
$$

The discriminant $1-\frac{40}{36}<0$, which implies that the equation has no solution. If $x_{3}=\frac{1}{2}\left(x_{1}+x_{2}\right)$, then the equation (4.2) equals with

$$
15 x_{1}^{2}-34 x_{1} x_{2}+15 x_{2}^{2}=0 .
$$

The discriminant $34^{2}-30^{2}=16^{2}>0$, which implies that the equation has two solutions. 
That is, the homogeneous manifold of type $E_{6}$-II in Theorem 3.16 admits exactly two invariant Einstein metrics up to proportionality. The parameters $\left(x_{1}, x_{2}, x_{3}\right)$ have the form $\left(\frac{5}{3} t, t, \frac{4}{3} t\right)$, or $\left(\frac{3}{5} t, t, \frac{4}{5} t\right)$, where $t>0$.

4.3. Invariant Einstein metrics on the three-locally-symmetric space of type $E_{6}$-III. For this case, $\mathfrak{h} \oplus \mathfrak{p}_{1}=A_{1} \oplus A_{5}$ and $\mathfrak{p}_{1} \subset A_{5}$. By the tables in [15], $\gamma_{1}=\frac{1}{2}$. From (4.1) and the dimensions in Table $2, \gamma_{2}=\frac{3}{4}$ and $\gamma_{3}=\frac{5}{12}$. It follows that

$$
a_{1}=\frac{1}{4}, \quad a_{2}=\frac{1}{8}, \quad a_{3}=\frac{7}{24} .
$$

Let $x_{1}=1$. The equations $r_{1}=r_{2}=r_{3}$ are equivalent to

$$
\left\{\begin{array}{l}
x_{2}^{2}-13 x_{3}^{2}+24 x_{2} x_{3}-24 x_{2}+13=0 \\
5 x_{2}^{2}-5 x_{3}^{2}+12 x_{3}-12 x_{2}+2=0 .
\end{array}\right.
$$

Excluding the summand containing $x_{2}^{2}$ form the first equation, we obtain

$$
x_{2}\left(40 x_{3}-36\right)=20 x_{3}^{2}+4 x_{3}-21 .
$$

For this case, $40 x_{3}-36 \neq 0$. Expressing $x_{2}$ by $x_{3}$ from (4.5) and inserting it into second one, we obtain

$$
1200 x_{3}^{4}-4960 x_{3}^{3}+7048 x_{3}^{2}-4152 x_{3}+855=0,
$$

which has two real solutions $x_{3} \approx 1.8845$ or $x_{3} \approx 0.4838$. From (4.5), $x_{2} \approx 1.4618$ or $x_{2} \approx 0.8640$.

That is, the homogeneous manifold of type $E_{6}$-III in Theorem 3.16 admits exactly two invariant Einstein metrics up to proportionality. The parameters $\left(x_{1}, x_{2}, x_{3}\right) \approx(t, 1.4618 t, 1.8845 t)$ or $\left(x_{1}, x_{2}, x_{3}\right) \approx(t, 0.8640 t, 0.4838 t)$, where $t>0$.

4.4. Invariant Einstein metrics on the three-locally-symmetric space of type $E_{7}-I$. For this case, $\mathfrak{h} \oplus \mathfrak{p}_{1}=A_{1} \oplus D_{6}$ and $\mathfrak{p}_{1} \subset D_{6}$. By the tables in [15], $\gamma_{1}=\frac{5}{9}$. From (4.1) and the dimensions in Table $2, \gamma_{2}=\gamma_{3}=\frac{5}{9}$. It follows that

$$
a_{1}=a_{2}=a_{3}=\frac{2}{9} \text {. }
$$

By Theorem 4.1, i.e. Theorem 3 in [23, the homogeneous manifold of type $E_{7}-\mathrm{I}$ in Theorem 3.16 admits exactly four invariant Einstein metrics up to proportionality. The parameters $\left(x_{1}, x_{2}, x_{3}\right)$ have the form $(t, t, t),\left(\frac{5}{9} t, \frac{4}{9} t, \frac{4}{9} t\right),\left(\frac{4}{9} t, \frac{5}{9} t, \frac{4}{9} t\right)$, or $\left(\frac{4}{9} t, \frac{4}{9}, \frac{5}{9} t\right)$, where $t>0$.

4.5. Invariant Einstein metrics on the three-locally-symmetric space of type $E_{7}$-II. For this case, $\mathfrak{h} \oplus \mathfrak{p}_{2}=A_{1} \oplus D_{6}$ and $\mathfrak{p}_{2} \subset D_{6}$. By the tables in [15], $\gamma_{2}=\frac{5}{9}$. From (4.1) and the dimensions in Table $2, \gamma_{1}=\frac{4}{9}$ and $\gamma_{3}=\frac{2}{3}$. It follows that

$$
a_{1}=\frac{5}{18}, \quad a_{2}=\frac{2}{9}, \quad a_{3}=\frac{1}{6} .
$$

Let $x_{1}=1$. The equations $r_{1}=r_{2}=r_{3}$ are equivalent to

$$
\left\{\begin{array}{l}
x_{2}^{2}+4 x_{3}^{2}-9 x_{2} x_{3}+9 x_{2}-4=0, \\
7 x_{2}^{2}-7 x_{3}^{2}+18 x_{3}-18 x_{2}-1=0 .
\end{array}\right.
$$

Excluding the summand containing $x_{2}^{2}$ form the first equation, we obtain

$$
x_{2}\left(63 x_{3}-81\right)=35 x_{3}^{2}-18 x_{3}-27 .
$$

For this case, $63 x_{3}-81 \neq 0$. Expressing $x_{2}$ by $x_{3}$ from (4.6) and inserting it into second one, we obtain

$$
2744 x_{3}^{4}-13482 x_{3}^{3}+24732 x_{3}^{2}-19926 x_{3}+5832=0
$$

which has two real solutions $x_{3} \approx 1.5535$ or $x_{3} \approx 0.7302$. From (4.6), $x_{2} \approx 1.7489$ or $x_{2} \approx 0.6139$. 
That is, the homogeneous manifold of type $E_{7}$-II in Theorem 3.16 admits exactly two invariant Einstein metrics up to proportionality. The parameters $\left(x_{1}, x_{2}, x_{3}\right) \approx(t, 1.7489 t, 1.5535 t)$ or $\left(x_{1}, x_{2}, x_{3}\right) \approx(t, 0.6139 t, 0.7302 t)$, where $t>0$.

4.6. Invariant Einstein metrics on the three-locally-symmetric space of type $E_{7}$-III. For this case, $\mathfrak{h} \oplus \mathfrak{p}_{1}=A_{7}$. It is the same as that of type $E_{7}$-II. That is, $\gamma_{1}=\frac{4}{9}$. From (4.1) and the dimensions in Table $2, \gamma_{2}=\gamma_{3}=\frac{4}{9}$. It follows that

$$
a_{1}=a_{2}=a_{3}=\frac{5}{18} \text {. }
$$

By Theorem 4.1, i.e. Theorem 3 in [23], the homogeneous manifold of type $E_{7}$-III in Theorem 3.16 admits exactly four invariant Einstein metrics up to proportionality. The parameters $\left(x_{1}, x_{2}, x_{3}\right)$ have the form $(t, t, t),\left(\frac{4}{9} t, \frac{5}{9} t, \frac{5}{9} t\right),\left(\frac{5}{9} t, \frac{4}{9} t, \frac{5}{9} t\right)$, or $\left(\frac{5}{9} t, \frac{5}{9} t, \frac{4}{9} t\right)$, where $t>0$.

4.7. Invariant Einstein metrics on the three-locally-symmetric space of type $E_{8}$-I. For this case, $\mathfrak{h} \oplus \mathfrak{p}_{2}=A_{1} \oplus E_{7}$ and $\mathfrak{p}_{2} \subset E_{7}$. By the tables in [15], $\gamma_{2}=\frac{3}{5}$. From (4.1) and the dimensions in Table $2, \gamma_{1}=\frac{7}{15}$ and $\gamma_{3}=\frac{3}{5}$. It follows that

$$
a_{1}=\frac{4}{15}, \quad a_{2}=a_{3}=\frac{1}{5} .
$$

If $x_{2}=x_{3}$, then the equation (4.2) is

$$
\frac{7}{15} x_{2}^{2}-x_{1} x_{2}+\frac{7}{15} x_{1}^{2}=0
$$

The discriminant $1-\left(\frac{14}{15}\right)^{2}>0$, which implies that the equation has two solutions. If $x_{1}=$ $\frac{2}{5}\left(x_{2}+x_{3}\right)$, then the equation (4.2) equals with

$$
147 x_{2}^{2}-281 x_{2} x_{3}+147 x_{3}^{2}=0 .
$$

The discriminant $281^{2}-294^{2}<0$, which implies that the equation has no solution.

That is, the homogeneous manifold of type $E_{8}$-I in Theorem 3.16 admits exactly two invariant Einstein metrics up to proportionality. The parameters $\left(x_{1}, x_{2}, x_{3}\right)$ have the form $(q t, t, t)$, where $t>0$ and $q$ is the root of the equation $7 x^{2}-15 x+7=0$.

4.8. Invariant Einstein metrics on the three-locally-symmetric space of type $E_{8}$-II. For this case, $\mathfrak{h} \oplus \mathfrak{p}_{1}=D_{8}$. It is the same as that of type $E_{8}$-I. That is, $\gamma_{1}=\frac{7}{15}$. From (4.1) and the dimensions in Table $2, \gamma_{2}=\gamma_{3}=\frac{7}{15}$. It follows that

$$
a_{1}=a_{2}=a_{3}=\frac{4}{15} .
$$

By Theorem 4.1, i.e. Theorem 3 in [23], the homogeneous manifold of type $E_{8}$-II in Theorem 3.16 admits exactly four invariant Einstein metrics up to proportionality. The parameters $\left(x_{1}, x_{2}, x_{3}\right)$ have the form $(t, t, t),\left(\frac{7}{15} t, \frac{8}{15} t, \frac{8}{15} t\right),\left(\frac{8}{15} t, \frac{7}{15} t, \frac{8}{15} t\right)$, or $\left(\frac{8}{15} t, \frac{8}{15} t, \frac{7}{15} t\right)$, where $t>0$.

4.9. Invariant Einstein metrics on the three-locally-symmetric space of type $F_{4}$-II. For this case, $\mathfrak{h} \oplus \mathfrak{p}_{1}=D_{4}$. By the tables in [15], $\gamma_{1}=\frac{7}{9}$. From (4.1) and the dimensions in Table $2, \gamma_{2}=\gamma_{3}=\frac{4}{9}$. It follows that

$$
a_{1}=\frac{1}{9}, \quad a_{2}=a_{3}=\frac{5}{18} .
$$

If $x_{2}=x_{3}$, then the equation (4.2) is

$$
\frac{7}{9} x_{2}^{2}-x_{1} x_{2}+\frac{7}{18} x_{1}^{2}=0
$$


The discriminant $1-\frac{98}{81}<0$, which implies that the equation has no solution. There exists none Einstein metrics. If $x_{1}=\frac{5}{9}\left(x_{2}+x_{3}\right)$, then the equation (4.2) equals with

$$
196 x_{2}^{2}-499 x_{2} x_{3}+196 x_{3}^{2}=0 .
$$

The discriminant $499^{2}-392^{2}>0$, which implies that the equation has two solutions.

That is, the homogeneous manifold of type $F_{4}$-II in Theorem 3.16 admits exactly two invariant Einstein metrics up to proportionality. The parameters $\left(x_{1}, x_{2}, x_{3}\right)$ have the form $\left(\frac{5}{9}(q+1) t, q t, t\right)$, where $t>0$ and $q$ is the root of the equation $196 x^{2}-499 x+196=0$.

\section{ACKNowledgments}

This work is supported by National Natural Science Foundation of China (No. 11001133). The first author would like to thank Prof. J.A. Wolf for the helpful conversation and suggestions.

\section{REFERENCES}

[1] D. Alekseevsky, New invariant Einstein metrics on generalized flag manifolds, Trans. Amer. Math. Soc. 337 (1993), 981-995.

[2] D. Alekseevsky, I. Dotti and C. Ferraris, Homogeneous Ricci positive 5-manifolds, Pacific J. Math. 175 (1996), 1-12.

[3] D. Alekseevsky and B. Kimel'Fel'D, Structure of homogeneous Riemannian spaces with zero Ricci curvature, Functional Anal. Appl. 9 (1975), 97-102.

[4] A. Arvanitoyeorgos and I. Chrysilos, Invariant Einstein metrics on genelalized flag manifolds with two isotropy summands, J. Aust. Math. Soc. 90 (2011), 237-251.

[5] Y. Bahturin and M. Goze, $\mathbb{Z}_{2} \times \mathbb{Z}_{2}$-symmetric spaces, Pacific J. Math. 236 (2008), 1-21.

[6] M. Berger, Les espaces symétriques noncompacts, Ann. Sci. École Norm. Sup. (3) 74 (1957), 85-177.

[7] A. Besse, Einstein manifolds, Ergeb. Math. 10 (1987), Springer-Verlag, Berlin-Heidelberg.

[8] C. Böнm, Homogeneous Einstein metrics and simplicial complexes, J. Differetial Geom. 67 (2004), 79-165.

[9] C. Bӧнm and M.M. KerR, Low-dimensional homogeneous Einstein manifolds, Trans. Amer. Math. Soc. 358(4) (2006), 1455-1468.

[10] C. Böhm, M. WANG and W. Ziller, A variational approach for homogeneous Einstein metrics, Geom. Funct. Anal. 14 (2004), 681-733.

[11] Z. Chen and K. Liang, Classification of analytic involution pairs of Lie groups (in Chinese), Chinese Ann. Math. Ser. A 26 (2005), no. 5, 695-708; translation in Chinese J. Contemp. Math. 26 (2005), no. 4, 411-424 (2006).

[12] Z. Chen and K. LiAng, Non-naturally reductive Einstein metrics on the compact simple Lie group $F_{4}$, Ann. Glob. Anal. Geom. 46 (2014), 103-115.

[13] M.K. Chunn and J.S. Huang, Double Vogan diagrams and semisimple symmetric spaces, Trans. Amer. Math. Soc. 362 (2010), 1721-1750.

[14] J.E. D'Atri and H.K. Nickerson, Geodesic symmetries in spaces with special curvature tensors, J. Differetial Geom. 9 (1974), 252-262.

[15] J.E. D'Atri and W. Ziller, Naturally reductive metrics and Einstein metrics on compact Lie groups, Memoirs of Amer. Math. Soc. 215 (1979).

[16] G.W. Gibbons, H. Lü and C.N. Pope, Einstein metrics on group manifolds and cosets, J. Geom. Phys. 61 (2011), no. 5, 947-960.

[17] J. Heber, Noncompact homogeneous Einstein spaces, Invent. math. 133 (1998), 279-352.

[18] J.S. HuAng and J. Yu, Klein four-subgroups of Lie algebra automorphisms, Pacific J. Math. 262 (2013), 397-420.

[19] M. KerR, New examples of homogeneous Einstein metrics, Michigan J. Math. 45 (1998), 115-134.

[20] M. Kimura, Homogeneous Einstein metrics on certain Kahler C-spaces, Adv. Stud. Pure Math., 18 (1990), 303-320.

[21] A. Kollross, Exceptional $\mathbb{Z}_{2} \times \mathbb{Z}_{2}$-symmetric spaces, Pacific J. Math. 242 (2009), 113-130.

[22] J. LAuret, Einstein solvmanifolds are standard, Ann. of Math. (2) 172 (2010), no. 3, 1859-1877.

[23] A.M. Lomshakov, Yu.G. Nikonorov and E.V. Firsov, Invariant Einstein metrics on three-locallysymmetric spaces, Mat. Tr. 6 (2003), no. 2, 80-101; translation in Siberian Adv. Math. 14 (2004), no. 3, 43-62.

[24] K. MorI, Left invariant Einstein metrics on $S U(n)$ that are not naturally reductive, Master Thesis (in Japanese) Osaka University 1994, English translation Osaka University RPM 96-10 (preprint series) 1996. 
[25] A.H. Mujtaba, Homogeneous Einstein metrics on $S U(n)$, J. Geom. Phys. 62 (2012), no. 5, 976-980.

[26] Yu.G. Nikonorov, On a class of homogeneous compact Einstein manifolds, Sibirsk. Mat. Zh., 41 (2000), 200-205; translation in Siberian Math. J., 41 (2000), 168-172.

[27] Yu.G. Nikonorov, Classification of generalized Wallach spaces, preprint, arXiv: 1411.3131, 2014.

[28] Yu.G. Nikonorov, E.D. Rodionov and V.V. Slavski, Geometry of homogeneous Riemannian manifolds, J. Math. Sci. 146(6) (2007), 6313-6390.

[29] C.N. Pope, Homogeneous Einstein metrics on $S O(n)$, arXiv: 1001.2776, 2010.

[30] E.D. Rodionov, Einstein metrics on even-dimensional homogeneous spaces admitting a homogeneous Riemannian metric of positive sectional curvature, Siberian Math. J. 41 (1991), 168-172.

[31] A. Sagle, Some homogeneous Einstein manifolds, Nagoya Math. J. 39 (1970), 81-106.

[32] B.L. WaERden, van der algebra, Springer-Verlag, Berlin, etc.

[33] N.R. WAllach, Compact homognenous Riemannian manifolds with strictly positive curvature, Ann. Math. (2) (96) (1972), 277-295.

[34] M. WANG and W. ZiLler, Existence and non-existence of homogeneous Einstein metrics, Invent. Math. 84 (1986), 177-194.

[35] Z.D. YAn, Real semisimple Lie algebras (in chinese), Nankai University Press, Tianjin, 1998.

School of Mathematical Sciences and LPMC, Nankai University, Tianjin 300071, P.R. China

E-mail address: chenzhiqi@nankai.edu.cn

Institute of Mathematics and Physics, Central South University of Forestry and Technology, Changsha Hunan, 410004, P. R. China

E-mail address: kangyf@tju.edu.cn

School of Mathematical Sciences and LPMC, Nankai University, Tianjin 300071, P.R. China

E-mail address: liangke@nankai.edu.cn 